\title{
Provisional diagnostic criteria for polymyalgia rheumatica: moving beyond clinical intuition?
}

\author{
Robert Spiera, ${ }^{1}$ Rene Westhovens ${ }^{2}$
}

In 1964, US Supreme Court Justice Potter Stewart, a master of words, when trying to define hardcore pornography admitted, 'I could never succeed in intelligibly doing so, but I know it when I see it...' Polymyalgia rheumatica (PMR) has been in that sense the pornography of rheumatic diseases - a difficult to define syndrome of inflammatory pain and stiffness in older people, but one commonly encountered in practice, and not commonly missed by experienced clinicians.

The response to low doses of corticosteroids is often rapid, dramatic, and has been used by many clinicians as a feature that helps define the disease. No single clinical finding or laboratory abnormality is unique to this disorder, and although some aspects of cytokine abnormalities and histopathological findings in this disorder are understood, there is no single identifiable aetiopathogenesis. The rather recent history of PMR is one of many names and definitions, reflecting this uncertainty about the pathology and pathogenesis. $^{1}$

In this issue of Annals of the Rheumatic Diseases, Dasgupta and colleagues ${ }^{2}$ report provisional classification criteria for PMR. In a study jointly sponsored by the European League Against Rheumatism and the American College of Rheumatology the authors propose a set of 'provisional' classification criteria. These emerged as the result of a multiphase effort in which they initially developed candidate criteria through a systemic literature review, then a consensus process and wider survey. These criteria were then assessed by experts, and those that emerged as most widely agreed upon were reviewed by both rheumatologists and non-rheumatologists in a wider survey generating a

This article is published simultaneously in the April 2012 issue of Arthritis \& Rheumatism.

${ }^{1}$ Hospital for Special Surgery, New York, New York, USA 2Department of Rheumatology , Katholieke Universiteit Leuven, Leuven 3000, Belgium

Correspondence to Rene Westhovens, Department of Rheumatology, Katholieke Universiteit Leuven, Leuven 3000, Belgium; rene.westhovens@uzleuven.be number of criteria most widely accepted. They then evaluated the performance of these proposed criteria in a 6-month prospective cohort study of 125 patients with new-onset PMR and 169 non-PMR control patients whose clinical presentation included features that can mimic PMR. They assessed the accuracy of the initial diagnoses at 6-month follow-up and assessed the relative performance of the individual proposed criteria for discriminating PMR from other conditions.

As a prerequisite, based on consensus of experts, all patients with PMR had to be $\geq 50$ years old and have elevations of C-reactive protein (CRP) and/or the erythrocyte sedimentation rate (ESR) (although the extent of those elevations was not defined). The proposed criteria are found to have a $68 \%$ sensitivity and a $78 \%$ specificity for discriminating PMR patients from comparison controls. In an explorative substudy, the authors evaluated the use of ultrasound in a scoring algorithm for classifying patients with PMR and found a poor capacity for discriminating shoulder conditions in PMR and rheumatoid arthritis, the latter probably posing the most important differential diagnostic challenge in this age group. Moreover, the authors do not report anything on intra and interobserver variability of these assessments that probably would also hamper the use of this instrument. They published a small validation substudy a few years ago focusing on interobserver variability of shoulder and hip pathologies but only two PMR and two rheumatoid arthritis patients were compared. ${ }^{3}$

It is important to emphasise that these proposed criteria are provisional (meaning that they must ultimately be confirmed in additional prospective validation cohorts) and not diagnostic criteria. Classification criteria are useful for defining patient groups for clinical or epidemiological studies. They are not developed or intended to define diagnoses in clinical practice. This point seems particularly relevant in PMR, a syndrome that is common, eminently treatable, and most often initially encountered by and treated by primary care providers rather than rheumatologists. These criteria, however, will undoubtedly be looked at by clinicians in clinical practice, so their performance in terms of sensitivity and specificity must be well understood and considered before clinicians utilise them. Is the sensitivity of these criteria adequate for practising clinicians, recognising that if the criteria are adopted, a third of patients they confront with PMR would be doomed to unnecessary suffering, when low-dose corticosteroids could rapidly ameliorate their often agonising pain? Although the authors indicate the dangers of overdiagnosis of this condition because of the potential of overusing corticosteroids, when used judiciously in relatively low doses with appropriate attention to the management of co-morbidities, this therapy can have a very favourable risk-benefit profile. ${ }^{4}$

These criteria are therefore most useful in the context of clinical therapeutic trials in which the homogeneity of the treated population must be maximised, although also recognising that should therapeutic alternatives to corticosteroids ultimately be defined from such studies, the generalisability to the larger world of PMR 'in practice' becomes less certain. This limitation is true for all clinical trials with rigidly defined patient populations, and is a constant tension between homogeneity of trials and generalisability in practice.

The lack of emergence of corticosteroid responsiveness as a discriminating factor in this study is perhaps one of the features most notably discordant from what clinicians generally appreciate in practice. This corticosteroid responsiveness indeed had been included in two previous widely used sets of criteria, namely the Healey criteria as well as the Jones/ Hazleman diagnostic criteria for PMR. . $^{5}$ The rapid and often dramatic response to low doses of corticosteroids is striking in most patients, often with a response recognised within 1 day of diagnosis. Although that observation is anecdotal, it is widely recognised as a feature that seems almost unique to PMR, and is different from what is generally appreciated in terms of response to therapy in other inflammatory musculoskeletal conditions. It is unclear from the protocol as reported whether the patients deemed not 'complete responders' were patients who had responded initially, but did not maintain that benefit at the 4-week follow-up, at which time disease activity was assessed (and by which point the corticosteroid dose had already been tapered). In practice, the lack of a dramatic and prompt 
response to low-dose corticosteroids would (and should) prompt a search for alternative diagnoses. It seems likely that part of the explanation of this high nonresponder rate is that the authors report this as a binary variable, namely recognising 'complete response' or lack thereof. It would be helpful to understand what constituted a complete response-would a patient with a near complete resolution of clinical symptoms and functional limitations but with persistence of some elevations of acute phase reactants be considered an 'incomplete responder' and therefore be included in that $30 \%$ ? In practice, such a response would probably be considered evidence in support of the diagnosis, but it would not have been captured as such in this study design.

It is also possible that multiple morbidities (such as concomitant underlying degenerative arthritis) confounded the interpretation of 'response' in this cohort, and that indeed is a common quandary in assessing disease activity in patients with PMR in clinical practice. Indeed, the criteria's requirement that pain be 'not better explained by other pathologies' is a major potential pitfall of the use of these criteria, both initially, and when judging response to therapy. The expertise and clinical judgement of the physician remain central to defining the presence of disease activity, and inasmuch make the reliability of these criteria subject to the expertise of the clinician.

Finally, the authors establish that age $\geq 50$ years and elevations of the ESR and/ or CRP were a requisite of the diagnosis. This seems appropriate for provisional classification criteria, in which the intent is to define a more homogeneous patient population for inclusion in clinical trials of potential targeted therapies that hope to afford a corticosteroid benefit. In practice, however, it is recognised that as many as $20 \%$ of patients have fairly classic PMR in the absence of elevations of sedimentation rate and/or CRP, again limiting the generalisability of these criteria for use in clinical practice. ${ }^{7}$ Indeed, it is those very patients without significant elevations of acute phase reactants in whom the spectacular response to low doses of corticosteroids can often be a most helpful feature diagnostically. The use of inflammation markers as dichotomous variables in the present classification criteria is an issue that will be debated in the future as there is a clear difference between an ESR above 100 compared with 30 in the minds of those who are going to use these criteria. Moreover, it seems that what are considered 'normal' values for ESR can differ based on age. ${ }^{8}$

Ultimately, although there are limitations in these provisional criteria, they are helpful as a framework for approaching diagnosis of this disease. It is certain that with better understanding of the underlying pathogenesis of PMR, trials of targeted therapies will probably be conducted that demand a unifying, less qualitative approach to the diagnosis. Moreover, a better understanding of pathogenesis could ultimately lead to the recognition of more specific biomarkers, which could make the need for such criteria less pressing. It is clear that the recognition of granulomatosis with polyangiitis (Wegener's granulomatosis) has been enhanced by the recognition of antineutrophil cytoplasmic antibodies in a majority of patients, particularly those with more diffuse forms of the disease.

The criteria proposed by Dasgupta and colleagues ${ }^{2}$ must therefore be recognised as what they are, namely provisional classification and not diagnostic criteria. These criteria cannot currently be compared to a 'gold standard' but they might facilitate future research to identify such specific disease markers. In future validation cohort studies, the performance of these criteria could even be compared with other widely used criteria such as the criteria of Bird et a 9 or Healey, ${ }^{5}$ and such research will contribute to a critical appraisal and their validation. Validation in comparison with control groups with more patients with different inflammatory and non-inflammatory pathologies coming also from other settings such as general medicine practices, and with longer than 6 months' follow-up will be helpful. Not surprisingly, given the somewhat vague nature of the syndrome, and the absence of a more specific biomarker, these criteria seem to have more modest sensitivity and specificity than was the case for many other rheumatic diseases. It seems clear that these criteria do not have adequate specificity or sensitivity to be applied to the approach of patients in the clinic, nor were they intended for such use. It is likely, however, that once published, such provisional criteria will be considered for use in practice by rheumatologists but even more so by primary care providers, who indeed are the ones most often diagnosing and even caring for patients with PMR. Although these criteria afford clinicians a basis for more objectively examining their diagnosis, and while allowing for a greater precision than Potter Stewart's definition of pornography, they cannot yet be assured to supercede the importance of clinical sense to which that rather vague definition speaks.

\section{Competing interests None}

Provenance and peer review Commissioned; externally peer reviewed.

Received 30 November 2011

Accepted 8 January 2012

Ann Rheum Dis 2012;11:475-476.

doi:10.1136/annrheumdis-2011-201111

\section{REFERENCES}

1. Hunder GG. The early history of giant cell arteritis and polymyalgia rheumatica: first descriptions to 1970. Mayo Clin Proc 2006;81:1071-83.

2. Dasgupta B, Cimmino MA, Maradit-Kremers H et al. 2012 provisional classification criteria for polymyalgia rheumatica: a European League Against Rheumatism/American College of Rheumatology collaborative initiative. Ann Rheum Dis 2012;71:484-92.

3. Scheel AK, Matteson EL, Dasgupta B, et al. Reliability exercise for the polymyalgia rheumatica classification criteria study: the oranjewoud ultrasound substudy. Int J Rheumatol 2009;2009:738931.

4. Hoes JN, Jacobs JW, Boers M, et al. EULAR evidence-based recommendations on the management of systemic glucocorticoid therapy in rheumatic diseases. Ann Rheum Dis 2007:66:1560-7.

5. Healey LA. Long-term follow-up of polymyalgia rheumatica: evidence for synovitis. Semin Arthritis Rheum 1984;13:322-8

6. Jones JG, Hazleman BL. Prognosis and management of polymyalgia rheumatica. Ann Rheum Dis 1981;40:1-5.

7. Helfgott SM, Kieval RI. Polymyalgia rheumatica in patients with a normal erythrocyte sedimentation rate. Arthritis Rheum 1996;39:304-7.

8. Osei-Bimpong A, Meek JH, Lewis SM. ESR or CRP? A comparison of their clinical utility. Hematology 2007:12:353-7.

9. Bird HA, Esselinckx W, Dixon AS, et al. An evaluation of criteria for polymyalgia rheumatica. Ann Rheum Dis 1979;38:434-9. 